\title{
Uniform acquisition modelling across PET imaging systems: unified scatter modelling
}

\author{
P. J. Markiewicz, M. J. Ehrhardt, D. Atkinson, S. R. Arridge, B. F. Hutton and S. Ourselin
}

1) Introduction: PET imaging plays an important role in understanding the biochemical underpinning of many diseases, for example the different phenotypes of dementia. The UK government launched a multi-million pound platform (the Dementias Platform UK) to facilitate one of the world's largest dementia population studies across multiple national centres equipped with the state-of-art PET/MR scanners, i.e., the Siemens Biograph mMR and GE Signa. However, the two scanners use different PET technologies (time of flight (TOF), silicon photomultiplier detectors (SiPM) versus nonTOF, avalanche photo-diodes (APD) detectors) which make the standardisation of image reconstruction necessary to obtain high quality datasets across different centres participating in the dementia platform [1]. One of the key aspects in high accuracy quantitative image reconstruction is correction for scatter events, and therefore, in this work we address one of the key reconstruction components-the scatter correction. Here we propose a novel approach to unified fully 3D TOF scatter modelling which inherently accounts for the difference in detection technologies as well as the large axial field of view (FOV) which is around $26 \mathrm{~cm}$ for both scanners. The key difference of the proposed model to the existing methods (e.g., [2], [3]), which use a line of response (LOR) driven approach, is that sin our model we adapt a voxel-based approach where each emission voxel is treated independently and for each of which a separate 3D probability scatter sinogram is found.

2) Methods: Consider an emission point within a brain, from which a photon pair is emitted in opposing directions $\hat{u}$ and $\hat{a}$ as shown in Fig. A. The photon travelling along $\hat{u}$ is assumed to get detected without scattering in the object, whereas the photon travelling along $\hat{a}$ is assumed to be scattered at any point along the path in such a way that it is detected by one of the detectors (this constitutes single scatter modelling). The fully 3D scatter distribution is found by considering all unscattered photon receiving crystals and finding a basic scatter distribution on the opposing side of the detector rings. This way, each crystal is considered to receive scattered and unscattered photons, but more importantly, the difference in distance travelled by both photons is inherently accounted for without additional calculations. Since the scatter distribution is smooth, only a limited set of crystal is considered, i.e., 64 out of 504 transaxial crystals in a single ring, and 8 out of 64 crystal rings (axially). This makes the axial and transaxial sampling roughly equal. This procedure is repeated for all the possible emission voxels. The model is implemented on the GPU using NVIDIA CUDA latest advances for fast reductions based on shuffle operations between multiple CUDA threads, and therefore, it allows significantly higher resolution images to be considered during the modelling $(117 \times 117 \times 63$ voxels as opposed to $32 \times 32 \times 27$ used by other methods). Full size scatter sinograms are found using intra-sinogram bi-cubic interpolation as well as bi-linear weighting performed in the Michelogram for inter-sinogram interpolation. Dedicated scatter normalisation efficiencies are found for each individual oblique sinogram (a total of 837 for span-11 and 4084 for span-1) using long acquisition (24-hour) phantom data. Since all the global proportions between scatter sinograms are maintained within the model, only a few scaling factors have to be obtained to fit the scatter distribution to the prompt data (127 factors out of 4084 for span-1). The model is applied to real data and also validated by a Monte Carlo simulation (a total of $30 \times 10^{9}$ events were simulated) using the Biograph mMR scanner geometry and SimSET for which the input (the $\mu$-map and emission image of ${ }^{18} \mathrm{~F}$-florbetapir) is shown in Fig. B.

3) Results: The key finding is presented in Fig. C where the method was validated with Monte Carlo simulations. It shows that for a given oblique sinogram of segments +3 and -3 for the non-TOF case there is little difference in scatter distribution (the top four plots; note the same value range for segment +3 and -3 sinograms). It would suggest that the popular $2 \mathrm{D}$ single scatter simulation can provide reasonably good estimates. However, this is no longer true for the TOF case (bottom four plots of Fig. C), where clear differences in the distributions (in shape and value range) are observed. The left plots of Fig. D show a sum of all TOF (bin 5) scatter sinograms in segment +3 in order to obtain better statistics for comparison with the Monte Carlo simulation. The right plots show sinogram profiles (as marked in the left plot) of the agreement between the Monte Carlo simulation and our model for non-TOF case (top plot) and TOF bin 5 (bottom plot). The scatter model is deployed in our stand-alone image reconstruction for the Siemens Biograph mMR, producing accurate estimates of scatter resulting in uniform image reconstruction as shown in Fig. E for a 24-hour cylindrical phantom acquisition. The 3 D TOF scatter probability distribution is obtained in 40 s on NVIDIA Tesla K20.

4) Conclusion: We have demonstrated a novel fully $3 \mathrm{D}$ scatter model which inherently accounts for TOF without additional computational cost, making the model useful for harmonised image reconstruction across different PET systems. In the case of TOF, the 3D nature of scatter emissions have to be considered, which is even more important in the case of whole body imaging.

\section{REFERENCES}

[1] Markiewicz, et al., "Verification of predicted robustness and accuracy of multivariate analysis", NeuroImage, vol. 56, 2011

[2] Watson et al. "Advances in Scatter Correction for 3D PET/CT", Nucl. Sci. Symp. Conf. Rec., 2004 IEEE

[3] Kim, et al., "Fully 3D iterative scatter-corrected OSEM for HRRT PET using a GPU", Phys. Med Biol., vol. 56, 2011 

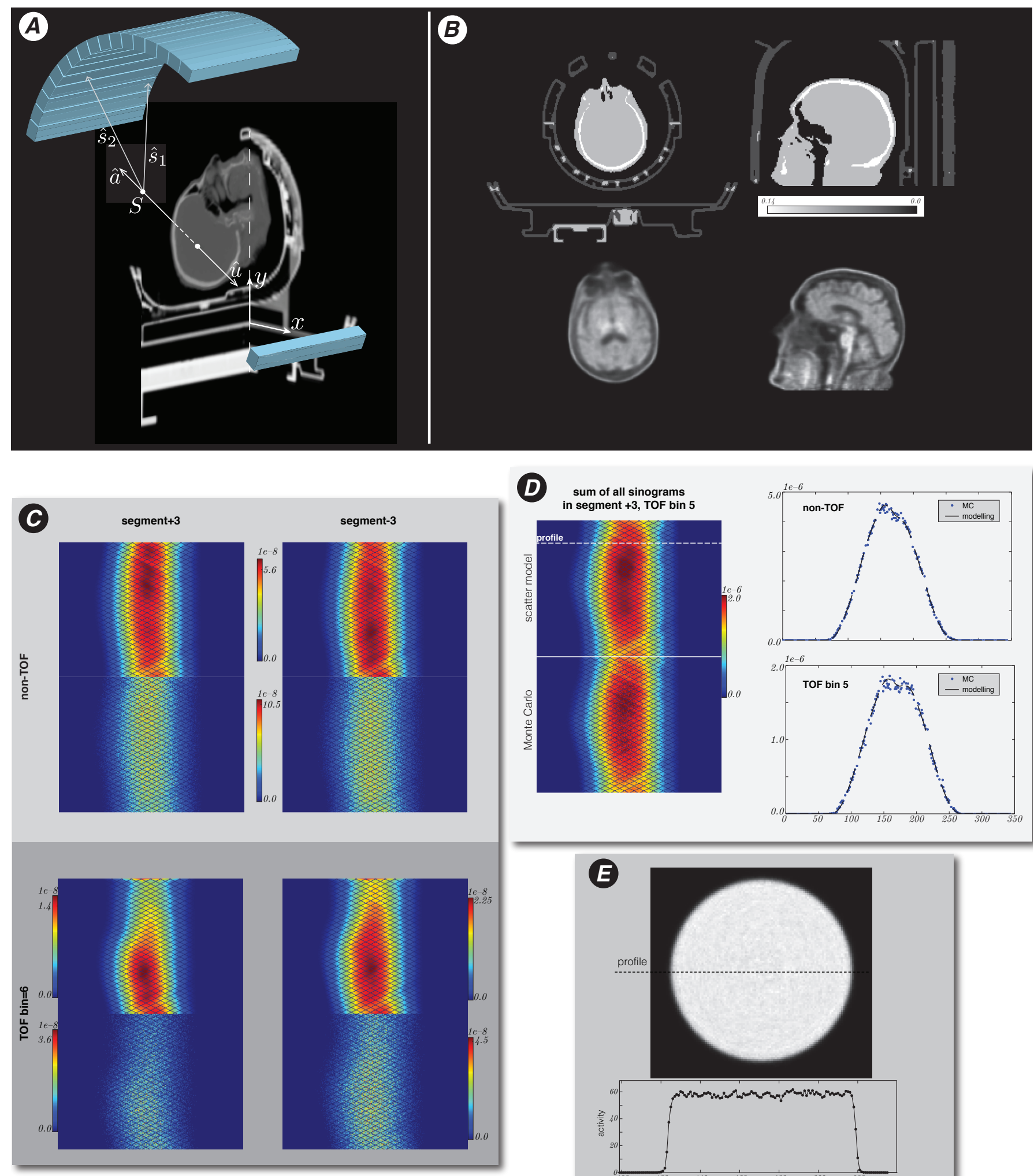

(D)
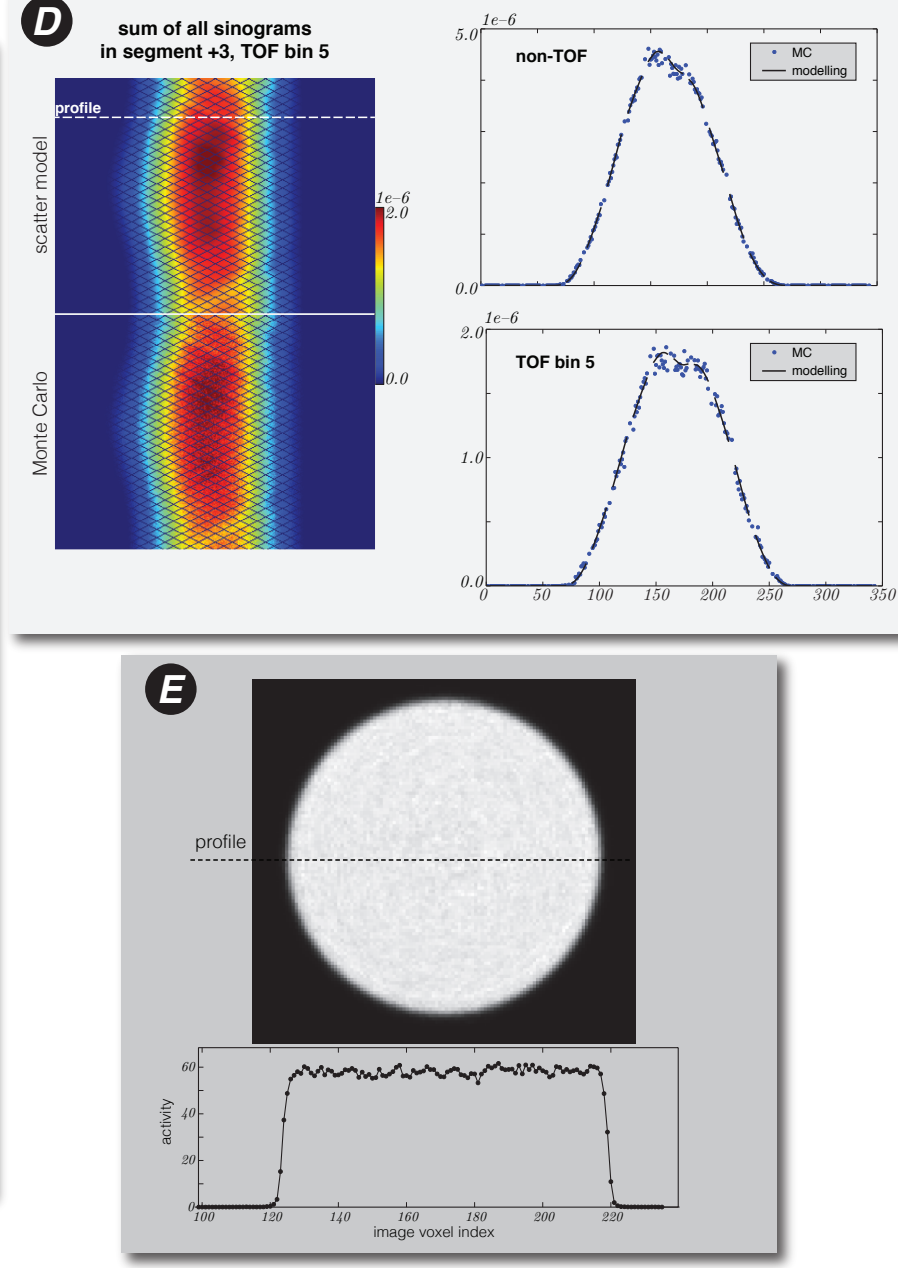

Fig. 1. A: A method of calculating fully 3D TOF scatter probability based on the $\mu$-map. From the emission point in the brain, the photon travelling in direction $\hat{u}$ is assumed unscattered, while the other photon travelling along $\hat{a}$ gets scattered at $S$ (any point along $\hat{a}$ ) at a scattering angle which makes the scattered photon hit the detector ring. B: transaxial and sagittal views of the $\mu$-map and emission image $\left({ }^{18} \mathbf{F}\right.$-florbetapir) used in Monte Carlo simulation . C: Estimated and Monte Carlo simulated oblique TOF and non-TOF scatter sinograms. Top four plots show one non-TOF sinogram at the same axial position but in segment +3 (left) and -3 (right), found using our model and Monte Carlo simulation. The bottom four plots show TOF sinogram for the middle bin (6) in segments +3 (left) and -3 (right), obtained by our model and Mote Carlo simulation. Note that there is greater difference between the sinograms in segments -3 and +3 for the TOF case than in the non-TOF case. D: Summed scatter sinograms in segment +3 for obtaining better statistics used in the comparison between our model and Monte Carlo simulation. Left plots show sum of all scatter sinograms for TOF bin 5, obtained by the model and the Monte Carlo simulation. The right plots show scatter sinogram profiles for the angle as shown in the left plot. The top profile plot is for non-TOF scatter sinograms summed in segment +3 , while the bottom sinogram profile is for the same sinogram angle but for TOF bin 5 . E: Image reconstruction of 24-hour cylindrical phantom acquisition. Top plot shows the transaxial slice reconstructed with out stand-alone software platform using the scatter model. Bottom plot shows the image profile, demonstrating the uniformity obtained by accounting for scatter (and other factors) accurately. 